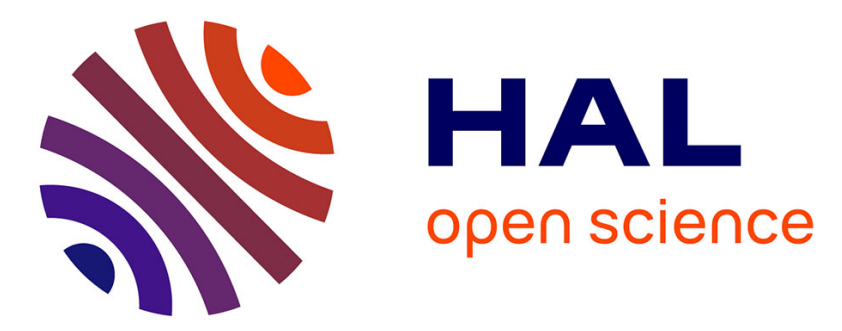

\title{
Length Sensing and Control in the Virgo Gravitational Wave Interferometer
}

\author{
F. Acernese, P. Amico, S. Aoudia, N. Arnaud, S. Avino, D. Babusci, G. \\ Ballardin, R. Barillé, F. Barone, L. Barsotti, et al.
}

\section{> To cite this version:}

F. Acernese, P. Amico, S. Aoudia, N. Arnaud, S. Avino, et al.. Length Sensing and Control in the Virgo Gravitational Wave Interferometer. IEEE Nuclear Science Symposium, Oct 2004, Rome, Italy. pp.1-6. in2p3-00025839

\section{HAL Id: in2p3-00025839 \\ https://hal.in2p3.fr/in2p3-00025839}

Submitted on 24 Mar 2006

HAL is a multi-disciplinary open access archive for the deposit and dissemination of scientific research documents, whether they are published or not. The documents may come from teaching and research institutions in France or abroad, or from public or private research centers.
L'archive ouverte pluridisciplinaire HAL, est destinée au dépôt et à la diffusion de documents scientifiques de niveau recherche, publiés ou non, émanant des établissements d'enseignement et de recherche français ou étrangers, des laboratoires publics ou privés. 


\section{Length Sensing and Control in the Virgo Gravitational Wave Interferometer}

F. Acernese ${ }^{\|}$, P. Amico ${ }^{x}$, S. Aoudia**, N. Arnaud ${ }^{\dagger \dagger}$, S. Avino" ${ }^{\|}$D. Babusci ${ }^{\S}$, G. Ballardin ${ }^{\dagger}$, R. Barillé ${ }^{\dagger}$, F. Barone ${ }^{\|}$, L. Barsotti ${ }^{\mathrm{xi}}$, M. Barsuglia ${ }^{\dagger \dagger}$, F. Beauville*, M.A. Bizouard ${ }^{\dagger \dagger}$, C. Boccara ${ }^{\ddagger \ddagger}$, F. Bondu** ${ }^{* *}$ L. Bosi ${ }^{\mathrm{x}}$, C. Bradaschia ${ }^{\mathrm{xi}}$, S. Braccini ${ }^{\mathrm{xi}}$, A. Brillet**, V. Brisson ${ }^{\dagger \dagger}$, L. Brocco ${ }^{\mathrm{xii}}$, D. Buskulic*, G. Calamai ${ }^{\ddagger}$, E. Callonill, E. Campagna ${ }^{\ddagger}$, F. Cavalier ${ }^{\dagger \dagger}$, R. Cavalieri ${ }^{\dagger}$, G. Cella ${ }^{\text {xi }}$, E. Chassande-Mottin**, F. Cleva**, J.-P. Coulon**, E. Cuoco ${ }^{\dagger}$, V. Dattilo ${ }^{\dagger}$, M. Davier ${ }^{\dagger \dagger}$, R. De Rosall, L. Di Fiore" , A. Di Virgilio ${ }^{\mathrm{xi}}$, B. Dujardin**, A. Eleuterill, D. Enard ${ }^{\dagger}$, I. Ferrante ${ }^{\mathrm{xi}}$, F. Fidecaro $^{\text {xi }}$, I. Fiori ${ }^{\text {xi }}$, R. Flaminio*, J.-D. Fournier**, S. Frasca ${ }^{\text {xii }}$, F. Frasconi ${ }^{\dagger}$, A. Freise ${ }^{\dagger}$, L. Gammaitoni ${ }^{\mathrm{x}}$, A. Gennai ${ }^{\text {xi }}$, A. Giazotto ${ }^{x i}$, G. Giordano ${ }^{\S}$, L. Giordano", R. Gouaty*, D. Grosjean*, G. Guidi ${ }^{\ddagger}$, S. Hebri**, H. Heitmann**, P. Hello ${ }^{\dagger \dagger}$, P. Heusse ${ }^{\dagger \dagger}$, L. Holloway ${ }^{\dagger}$, S. Kreckelbergh ${ }^{\dagger \dagger}$, P. La Penna ${ }^{\dagger}$, V. Loriette ${ }^{\ddagger \ddagger}$, M. Loupias ${ }^{\dagger}$, G. Losurdo ${ }^{\ddagger}$, J.-M. Mackowski ${ }^{\mp}$, E. Majorana ${ }^{\text {xii }}$, C. N. Man**, F. Marchesoni ${ }^{\text {, }}$, E. Marchetti ${ }^{\ddagger}$, F. Marion*, J. Marque ${ }^{\dagger}$, F. Martelli ${ }^{\ddagger}$, A. Masserot ${ }^{*}$, M. Mazzoni ${ }^{\ddagger}$, L. Milano $\|$, C. Moins ${ }^{\dagger}$, J. Moreau ${ }^{\ddagger \ddagger}$, N. Morgado ${ }^{\Uparrow}$, B. Mours ${ }^{*}$, J. Pacheco** ${ }^{* *}$ A. Pai ${ }^{\text {xii }}$, C. Palomba ${ }^{\text {xii }}$, F. Paoletti ${ }^{\dagger}$, S. Pardill, A. Pasqualetti ${ }^{\dagger}$, R. Passaquieti ${ }^{\text {xi }}$,

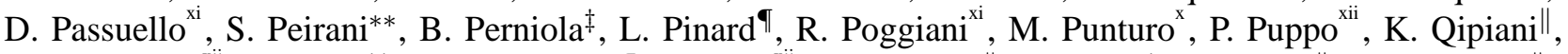

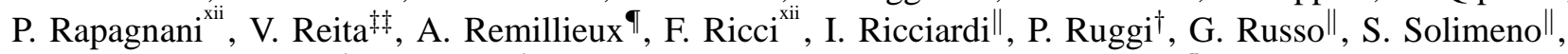
A. Spallicci**, R. Stanga ${ }^{\ddagger}$, R. Taddei ${ }^{\dagger}$, D. Tombolato*, E. Tournefier*, F. Travasso ${ }^{x}$, D. Verkindt*, F. Vetrano ${ }^{\ddagger}$, A. Viceré ${ }^{\ddagger}$, J.-Y. Vinet**, H. Vocca ${ }^{*}$, M. Yvert* and Z.Zhang ${ }^{\dagger}{ }^{*}$ Laboratoire d'Annecy-le-Vieux de Physique des Particules, Annecy-le-Vieux, France;

${ }^{\dagger}$ European Gravitational Observatory (EGO), Cascina (Pi), Italia;

${ }^{\ddagger}$ INFN, Sezione di Firenze/Urbino, Sesto Fiorentino, and/or Università di Firenze, and/or Osservatorio Astrofisico di Arcetri, Firenze and/or Università di Urbino, Italia;

$\S$ INFN, Laboratori Nazionali di Frascati, Frascati (Rm), Italia;

"SMA, IPNL, Villeurbanne, Lyon, France;

"INFN, sezione di Napoli and/or Università di Napoli "Federico II" Complesso Universitario di Monte S.Angelo, and/or Università di Salerno, Fisciano (Sa), Italia;

** Departement Artemis - Observatoire de la Côte d'Azur, BP 4220906304 Nice, Cedex 4, France;

${ }^{\dagger \dagger}$ Laboratoire de l'Accélérateur Linéaire (LAL), IN2P3/CNRS-Univ. de Paris-Sud, Orsay, France;

${ }_{\ddagger}^{\ddagger}$ ESPCI, Paris, France;

${ }^{x}$ INFN, Sezione di Perugia and/or Università di Perugia, Perugia, Italia;

${ }^{x i}$ INFN, Sezione di Pisa and/or Università di Pisa, Pisa, Italia;

${ }^{x i i}$ INFN, Sezione di Roma and/or Università ”La Sapienza", Roma, Italia.

\begin{abstract}
The gravitational wave detector Virgo is presently being commissioned. A significant part of last year was spent in setting up the cavity length control system. This work was carried out with steps of increasing complexity: locking a simple FabryPerot cavity, then a Michelson interferometer with Fabry-Perot cavities in both arms, and finally recycling the light beam into the interferometer. The applied strategy and the main results obtained are described.
\end{abstract}

Index Terms-Gravitational waves, Interferometry, FabryPerot, Feedback control system.

\section{INTRODUCTION}

$\mathbf{T}$ HE Virgo interferometer aims to detect gravitational waves emitted by astrophysical sources [1] in a frequency range between few $\mathrm{Hz}$ and few $\mathrm{kHz}$. Virgo consists in a power recycled Michelson interferometer with $3 \mathrm{~km}$ Fabry-Perot cavities in the arms. Other detectors, such as LIGO [2], TAMA [3] and GEO [4] have a similar structure. In Virgo all the mirrors are suspended from the so called superattenuators (SA), multipendulum isolators able to reduce the seismic noise transferred to the instrument below the limits requested for the detection [5].

The maximum sensitivity of an interferometric detector like Virgo is achieved by selecting an appropriate working point:

- the laser light is resonant in the optical cavities, 
- the output port is tuned on the dark fringe.

These conditions translate into fixed relationships between the laser light wavelength $(1.064 \mu \mathrm{m})$ and the independent lengths of the ITF [6]. For a configuration like Virgo the laser frequency and four independent lengths have to be controlled (Fig. 1):

- the recycling cavity length $l_{r}=l_{0}+\frac{l_{1}+l_{2}}{2}$;

- the length asymmetry between the arms, or dark fringe (DF) length, $\Delta l=l_{1}-l_{2}$;

- the length of the two long arms, $L_{1}$ and $L_{2}$.

The process by which an interferometer (ITF) in an initially uncontrolled condition is brought and kept on its working point by closing the feedback loops is called lock acquisition.

\section{THE LOCK ACQUISITION PROBLEM}

The Virgo SA provide a very good seismic isolation in the frequency band used for detection, but below a few $\mathrm{Hz}$ some seismic noise is transferred to the mirrors or even amplified by the SA mechanical resonances. While the expected sensitivity is of the order of $10^{-18} \mathrm{~m} / \sqrt{\mathrm{Hz}}$, the allowed deviation from the working point, or locking accuracy, is $10^{-12} \mathrm{~m} \mathrm{rms}$. A feedback control system is needed to keep the ITF locked on the required interference conditions, acting on the mirrors without reintroducing noise in the detection band.

Relative motion of the mirrors is detected using a carrier beam phase modulated at $f=6.2641 \mathrm{MHz}$. The carrier senses the resonant response of the optical cavity; its beating with the sidebands produces light modulated in intensity with frequency $f, 2 f, \ldots$ collected by a photodiode. The demodulated signal contains the phase response of the cavity [7], [8] and therefore the length deviation from resonance. Appropriate positioning of the photodiodes allows to sense the various lengths involved. These error signals are digitized and all sent to the control system (Global Control [6]) which computes the corrections to be applied to the mirrors by the suspension actuators.

In order to test the stability and the robustness of all the subsystems involved in the operations and to get some experience on the lock acquisition, the Virgo commissioning activity was organized in steps of increasing complexity:

- the separate commissioning of the North and West FabryPerot cavities,

- the commissioning of the recombined Michelson FabryPerot ITF,

- the commissioning of the recycled Michelson Fabry-Perot ITF.

\section{LOCK OF A SINGLE FABRY-PEROT CAVITY}

The locking scheme of a single Fabry-Perot cavity is based on the classical Pound-Drever-Hall technique [7], [8], with the reflected demodulated signal (or with the transmitted demodulated signal) used as error signal [11]. As already successfully tested during the commissioning of the central interferometer [9], the lock is acquired using:
- an asymmetric trigger on the transmitted power, with an input threshold set at about half of the expected maximum power of the fundamental mode $\mathrm{TEM}_{00}$. In this way the $\mathrm{TEM}_{00}$ resonance can be isolated from higher order modes of the cavity. Moreover, by setting an output threshold at few percent of the maximum value, the time the feedback is active can be increased.

- the linearization of the error signals. The shape of the error signal is determined by the phase response and the light intensity in the cavity (DC signal). By correcting for the latter factor a linear signal is obtained over the width of the cavity resonance. (see Figure 3). This is essential to achieve sufficient duration of the feedback action.

Figure 2 shows the locking scheme used to control the North cavity: an equivalent scheme is applied to the West cavity. With this technique the lock of the North cavity, previously simulated [12] in the time domain, was acquired in a stable way at first trial.

\section{THE RECOMBINED INTERFEROMETER}

\section{A. Lock acquisition}

The recombined ITF is a Michelson with Fabry-Perot cavities in the arms. In this configuration there are three lengths to be controlled: $L_{1}, L_{2}$ and $\Delta l$. Using the end signals the two long arms can be controlled independently, acting on the corresponding end mirrors (Figure 4).

Once the cavities are locked, the Michelson length is controlled acting on the BS mirror, using the output port demodulated signal, or equivalently the reflected demodulated signal. With this strategy the lock acquisition is very fast, usually in few seconds the recombined ITF goes from an uncontrolled to a fully controlled state. An example of lock acquisition event is shown in Figure 5.

\section{B. Linear locking}

The end mirrors are almost totally reflective and the transmitted power is very low. The end photodiodes used in the lock acquisition phase are electronic noise limited giving too low a signal-to-noise ratio. Another set of signals, to be used in a linear regime to control the ITF, is needed.

Once the interferometer is locked the common mode of the arms $L_{1}+L_{2}$ is also given by the in phase demodulated component of the light reflected by the ITF (2_phase), as shown in Figure 6. The differential mode $L_{1}-L_{2}$ is given instead by the in phase output port light (1_demod), which is slightly contaminated by $\Delta l$, mainly provided by the other quadrature of the ITF reflected light (2_quad). The light intensity is higher giving a much better signal-to-noise ratio. Linear combinations of the error signals are computed to provide the correction forces to the mirrors. This phase is called linear locking.

\section{LOCK ACQUISITION OF THE RECYCLED INTERFEROMETER}

The strategy developed [13] and successfully adopted in the LIGO interferometer [14], which has a similar optical scheme, 
was taken as a starting point for the lock acquisition scheme of the recycled interferometer. A second technique, called variable finesse lock acquisition, has been recently developed and successfully tested.

\section{A. LIGO like technique}

In the LIGO technique the four degrees of freedom of the ITF are sequentially locked, dynamically changing the optical sensing matrix in order to compensate the variation of the fields in the course of lock acquisition. The final lock is reached in a sequence of 3 different steps, starting from the uncontrolled condition, where only the final one is stable:

- in the first step the sidebands enter in resonance in the recycling cavity (anti-resonant in the arms), the carrier is on the anti-resonance for the recycling cavity and for the arms (unstable state);

- in the second step the sidebands remains in the same condition, the carrier enter in resonance in one arm, on the bright fringe in the recycling cavity (unstable state);

- in the last step the carrier goes in resonance in both arms and in the recycling cavity (stable state).

The approach is statistical: the control loops for the 4 degrees of freedom are engaged every time the ITF passes through the relative resonance conditions, corresponding to the 3 different steps described above, until the sequence is completed.

This baseline technique has been experimentally tested on the Virgo ITF for about one month, starting the work with the lock of intermediate stable states:

- lock of the recycling cavity, with the arms end mirrors misaligned;

- lock of the recycling cavity plus one arm, with the end mirror of the other arm misaligned.

This preliminary activity was extremely important to check the robustness and the stability of all the ITF sub-systems and to develop ideas suited to the Virgo characteristics.

In this context the use of the reflected $3 f$-demodulated signal (used in the TAMA scheme [15]) to control the recycling cavity length had to be considered. From the analysis of the frequency response of the error signals, it emerges that the transfer functions for the reflected $f$-demodulated signal are strongly dependent on the ITF losses. A first evaluation shows that the resulting transfer functions make control very difficult. The reflected $3 \mathrm{f}$-demodulated signal, instead, is less sensitive to losses and the frequency response is almost constant. Besides, while the reconstruction of the recycling cavity length with the $f$-demodulated signal is critical because of the contamination of the common length of the cavities, the $3 f$-demodulated signal has much less contamination.

Some stable intermediate configurations of the ITF were locked with this technique, and some tests of a full locking were carried out. At the same time an alternative technique was tested, and it gave rapidly encouraging results.

\section{B. Variable finesse lock acquisition}

The basic idea is to lock the ITF outside the working point for the dark fringe. In this way a good fraction of light escapes through the output port and the power build-up in the recycling cavity is low. Then the ITF is brought adiabatically on the dark fringe. This technique has been called variable finesse, because the finesse of the recycling cavity is changed during the lock acquisition path.

The procedure starts with the PR mirror initially misaligned by some microradians. The simple Michelson is controlled on the half fringe, using the output port DC signal (1_DC), while the two arms are independently locked using the end photodiodes. The power recycling cavity length is robustly controlled using the reflected $3 \mathrm{f}$-demodulated signal, as previously described. In this way all the four degrees of freedom of the ITF are locked since the beginning of the lock acquisition in a stable way, preventing excitations of the mirrors. From this starting condition, the PR is realigned (fig 7), keeping always the Michelson on the half fringe, giving a very low recycling gain.

In order to increase the recycling gain the Michelson has to be brought on the dark fringe: this is done adiabatically, decreasing the offset in the Michelson error signal. At the same time, the control scheme changes. The end photodiodes can be used to control independently the cavities only when the ITF is on the half fringe: going to the dark fringe they start to be strongly coupled and a common and differential control has to be activated to keep the lock.

Then a frequency stabilization servo is engaged, controlling the common mode of the cavities with a very high bandwidth: the contamination by this degree of freedom on all the photodiodes is so cancelled.Tthe differential mode is kept locked by one of the end photodiode signals (fig 8).

The final step consists in switching from the DC to a demodulated signal (the signal used is 5_demod) to control $\Delta l$ (Figure 9): this is done in proximity of the dark fringe. Eventually the offset in the Michelson error signal is removed, the ITF goes on the dark fringe and the recycling cavity gain increases up to the maximum value.

Using this technique the lock acquisition of the full Virgo ITF was reached, as shown in Figure 10, and the recycling gain achieved was about 25 . The analysis of this lock acquisition procedure is currently in progress.

\section{CONCLUSION}

The work in order to control the Virgo ITF was presented. After a first study of the Fabry-Perot cavities a large amount of time was spent on the recombined ITF. To lock the recycled interferometer two techniques have been presented: the first one, based on the LIGO experience, and the variable finesse one, which allowed acquisition of the lock of the full Virgo detector.

\section{REFERENCES}

[1] C. Bradaschia et al., Nuclear Instruments and Methods in Physics Research A 289, 518-525 (1990). 
[2] D. Sigg et al., "Commissioning of the LIGO detectors",Class.Quant.Grav., 19(7), 1429-1435 (2002).

[3] M.Ando et al., Current status of TAMA, Class.Quant.Grav. 19(7), 1409$1419,(2002)$.

[4] B. Willke et al., The GEO 600 gravitational wave detector, Class.Quant.Grav., 19(7), 1377-1387 (2002).

[5] G.Ballardin et al., Measurement of the VIRGO superattenuator performance for seismic noise suppression, Rev. Sci. Instrum. 72 (2001) 36433652

[6] F.Cavalier, Le controle global de Virgo, These d'Habilitation a diriger des Recherches, Universitè de Paris Sud, LAL 01-69 (2001).

[7] R.V.Pound, Electronic Frequency Stabilization of Microwave Oscillators, Rev. Sci. Instrum. 17 (1946) 490-505

[8] R.W.P.Drever et al., Laser phase and frequency stabilization using an optical resonator, Appl. Phys. B: Photophys. Laser Chem. 31 (1983) 97105

[9] M.Barsuglia et al., Lock Acquisition of the central interferometer of the gravitational wave detector Virgo, Astroparticle Physics 21 (2004) 465477

[10] F.Acernese et al., Commissioning the central interferometer of the gravitational wave detector Virgo, Astroparticle Physics 21 (2004) 1-22

[11] R.Flaminio et al., The commissioning of the Virgo Interferometer Arms, Virgo Note VIR-NOT-LAP-1390-266

[12] B.Caron, L.Derome, R.Flaminio, X.Grave, F.Marion, B.Mours, D.Verkindt, F.Cavalier and A.Viceré, SIESTA, a time domain, general purpose simulation program for the VIRGO experiment, Astroparticle Physics 10 (1999) 369-386.

[13] M.Evans, Lock acquisition in Resonant Optical Interferometers, $\mathrm{PhD}$ Thesis, CALTECH (2001)

[14] M.Evans et al., Lock acquisition of a gravitational-wave interferometer, OPTICS LETTERS vol.27, n.8. April 15, (2002)

[15] K.Arai and the TAMA collaboration Sensing and controls for powerrecycling of TAMA300, submitted to Class. Quantum Grav.

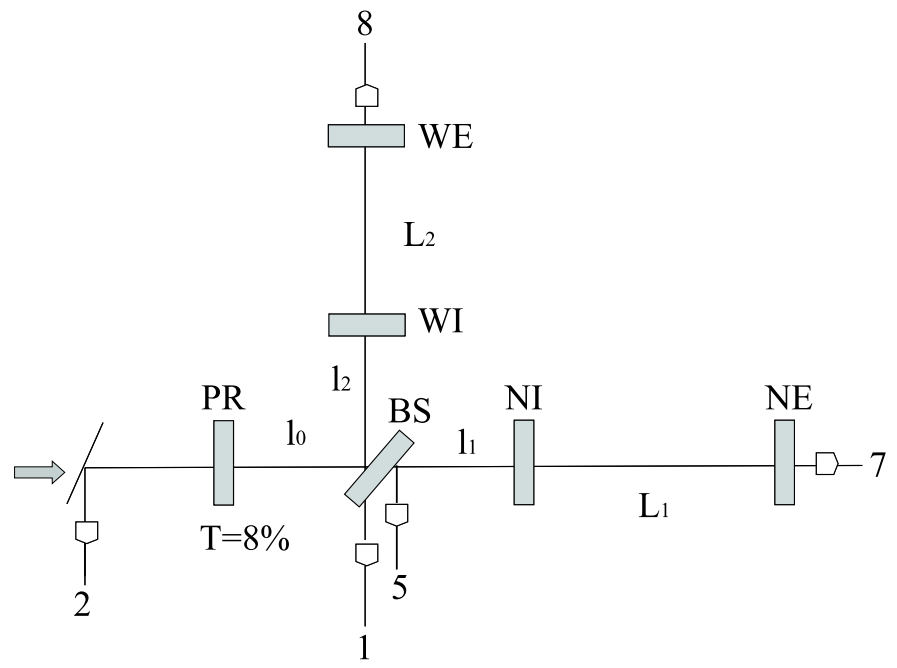

Fig. 1. Optical scheme of the Virgo interferometer: the finesse of the long arms is 50. In the picture are indicated the mirrors, the lengths of the interferometer and the photodiodes used during the lock acquisition.

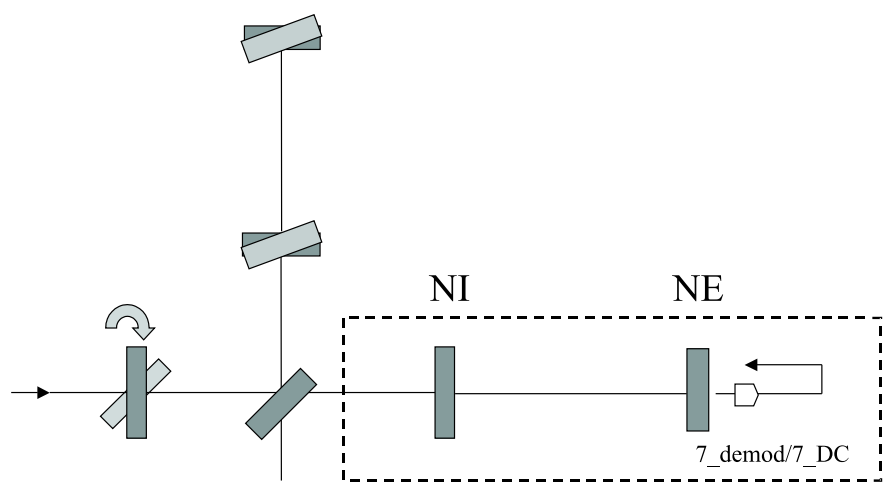

Fig. 2. Lock acquisition scheme of the North Fabry-Perot cavity, using the transmitted demodulated signal as error signal. The PR mirror, as well as the west arm, are misaligned. 

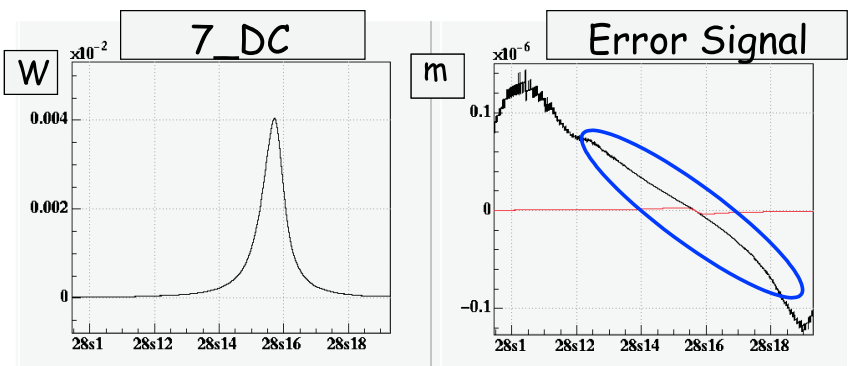

Fig. 3. Effect of the normalization of the error signal: the right plot shows the error signal with and without the normalization by the transmitted power (resonance peak shown in the left plot). Note that by normalization the linear zone (inside the ellipse) of the error signal increases of about one order of magnitude.

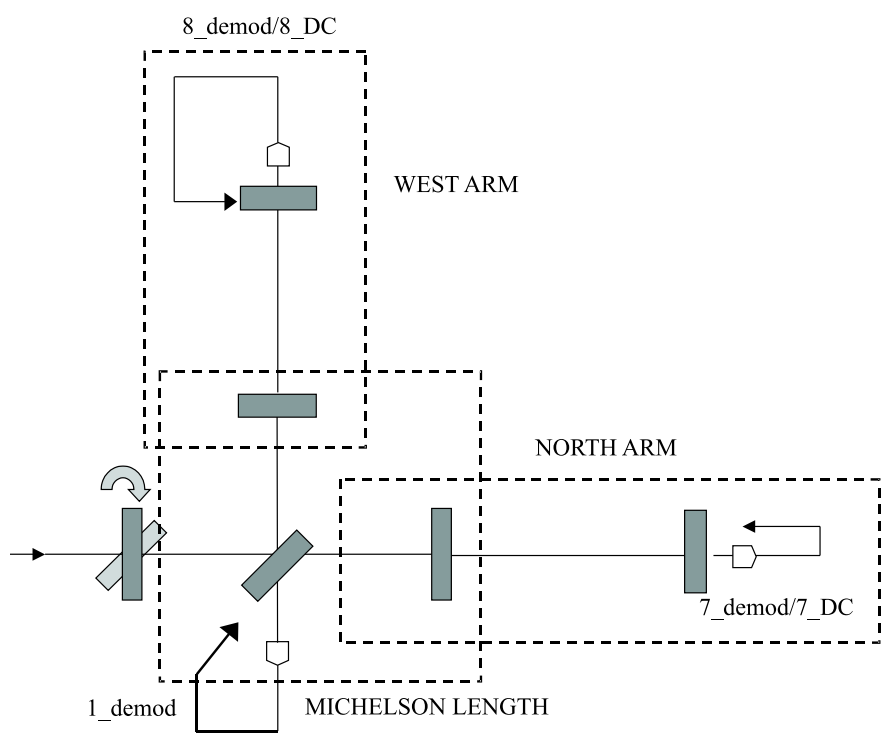

Fig. 4. Lock acquisition scheme of the recombined ITF: the three independent lengths are controlled using the signals of the end photodiodes and the output port demodulated signal.

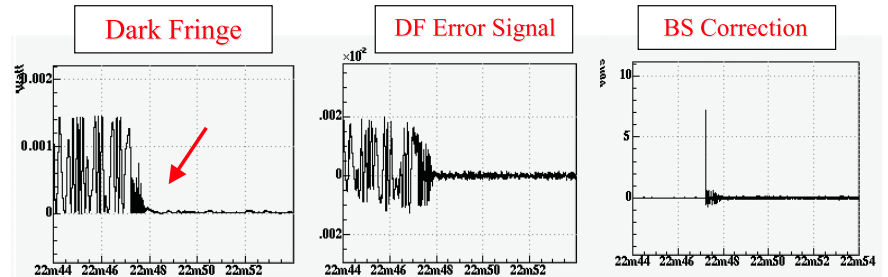

Fig. 5. Lock acquisition of the recombined ITF: when the Fabry-Perot cavities are locked and the dark fringe power goes down a given threshold, usually half of the maximum power, the trigger is engaged and the corrections start to be sent to the BS, in order to lock the Michelson length. The first plot shows the dark fringe power, the second one the error signal to control the Michelson length, the third one the corrections sent to the BS mirror.

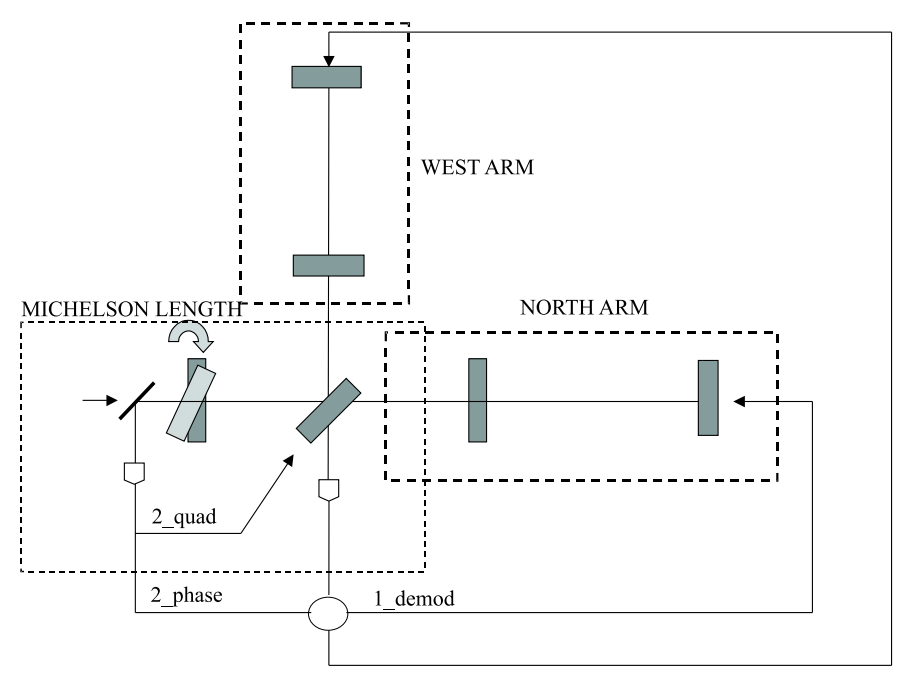

Fig. 6. Linear locking of the recombined ITF: the end photodiodes signals are replaced with the reflected and the output port one, because of noise reasons.

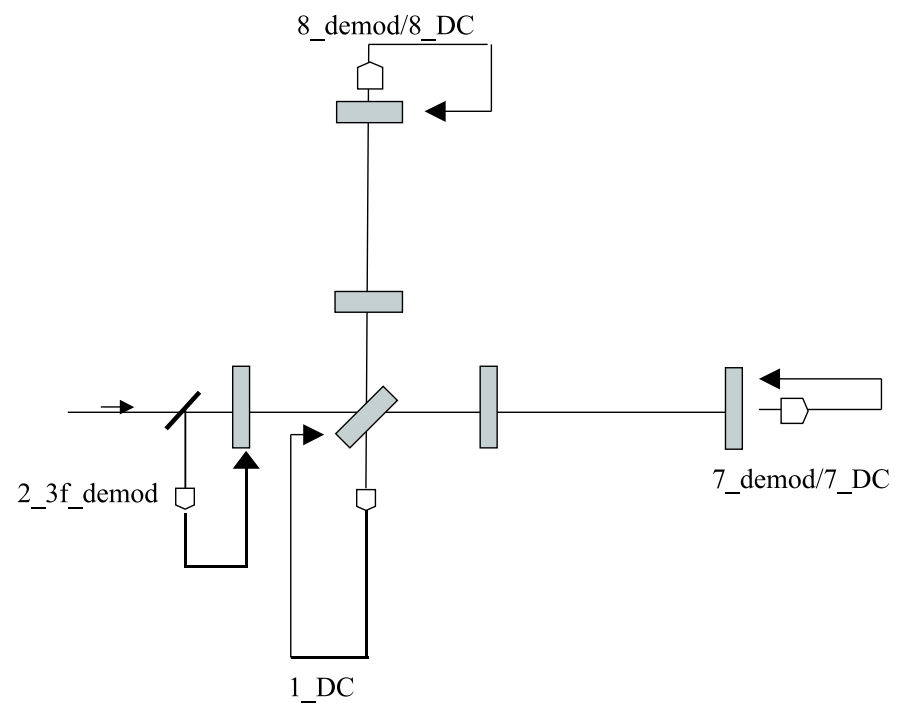

Fig. 7. First step of the lock of the recycled configuration, after the realigning of the PR mirror: all the four degrees of freedom are locked since the beginning. 


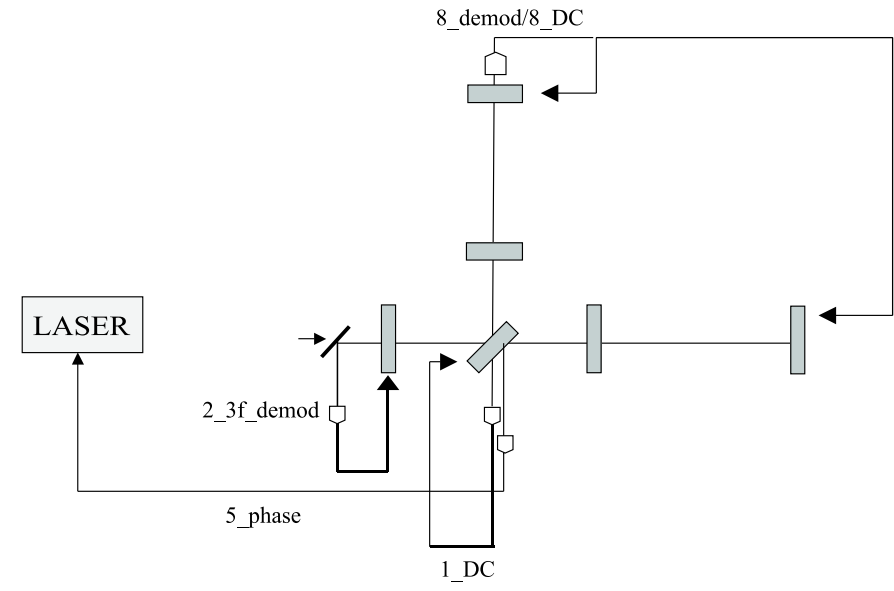

Fig. 8. Second step of the lock of the recycled configuration: going to the dark fringe of the Michelson, the frequency stabilization servo is engaged to control the common mode of the arms, one of the two end photodiodes signals is switched off and the other one is used to control the differential mode.

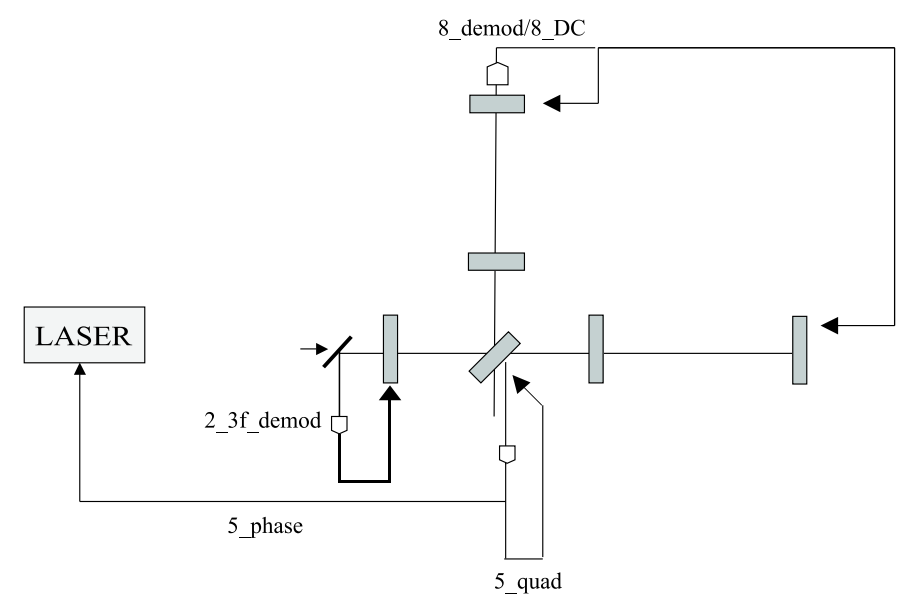

Fig. 9. Third step of the lock of the recycled configuration: near to the dark fringe, the error signal of the Michelson is switched from a DC to a demodulated one. Than the offset in the error signal is completely reduced to 0 .
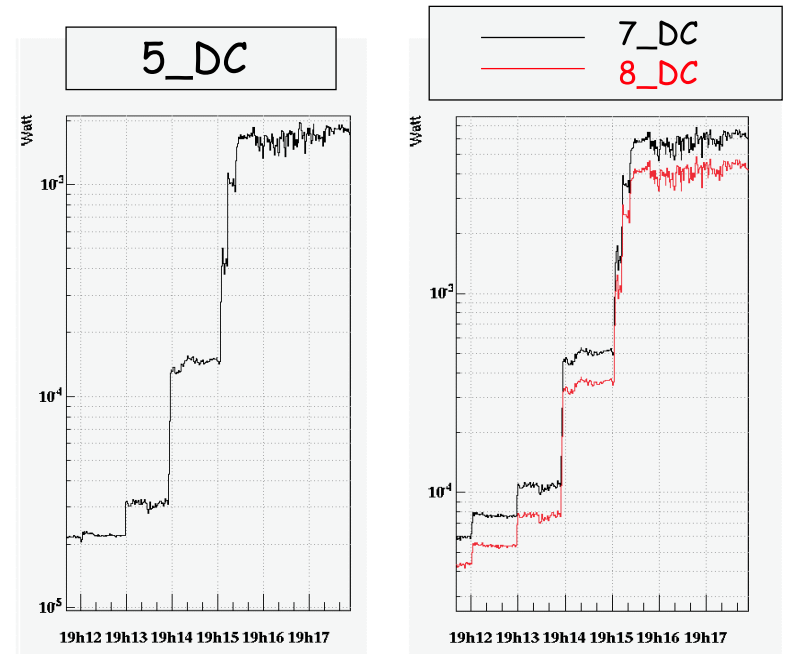

19h12 19h13 19h14 19h1519h16 19h17

Fig. 10. Lock acquisition event with the variable finesse technique: the left plot shows the recycling cavity power, measured by the light reflected by the second face of the beam-splitter mirror, the right plot shows the powers transmitted from the north and the west cavities. 